\title{
Preterm children's long-term academic performance after adaptive computerized training: A randomized controlled trial
}

Julia Jaekel ${ }^{2} 3$, Katharina Heuser ${ }^{3}$, Gisela Axmacher-Bopp ${ }^{3}$, Claudia Roll ${ }^{4}$, Dariusz Michna ${ }^{5}$, Thomas Hoehn ${ }^{6}$, Norbert Teig $^{7}$, Francisco Brevis ${ }^{8}$, Antonia Zapf ${ }^{9}$, Peter Bartmann ${ }^{10}$, Dieter Wolke ${ }^{2}$, Úrsula Felderhoff-Mueser ${ }^{11}$, \& Britta Huening $^{11}$ - nothing to disclose -

\section{Background and aims}

Preterm children often struggle in school because of working memory and mathematic difficulties. Adaptive computerized interventions may help improve academic success but randomized trials for school-aged preterm children are rare. We tested whether adaptive online math training (XtraMath(C) versus working memory training (Cogmed(C) improves academic performance.

\section{Methods}

During their first year of elementary school, N=65 preterm children (28 $35+6$ weeks gestation; stratified for gestational age and gender) were recruited into a prospective multi-center trial (Figure 1). Participants were randomized into two groups and received one of two computerized trainings at home for five weeks.

Primary Outcome: Teachers rated academic performance (TAAS) in math, reading, and attention compared to classmates before (pretest), directly after (posttest), and 12 months after the intervention (follow-up). Total academic growth (change from baseline) was calculated as z-standardized difference. Math tests were administered as secondary outcomes.

\section{Figure 1. Randomization flow chart}

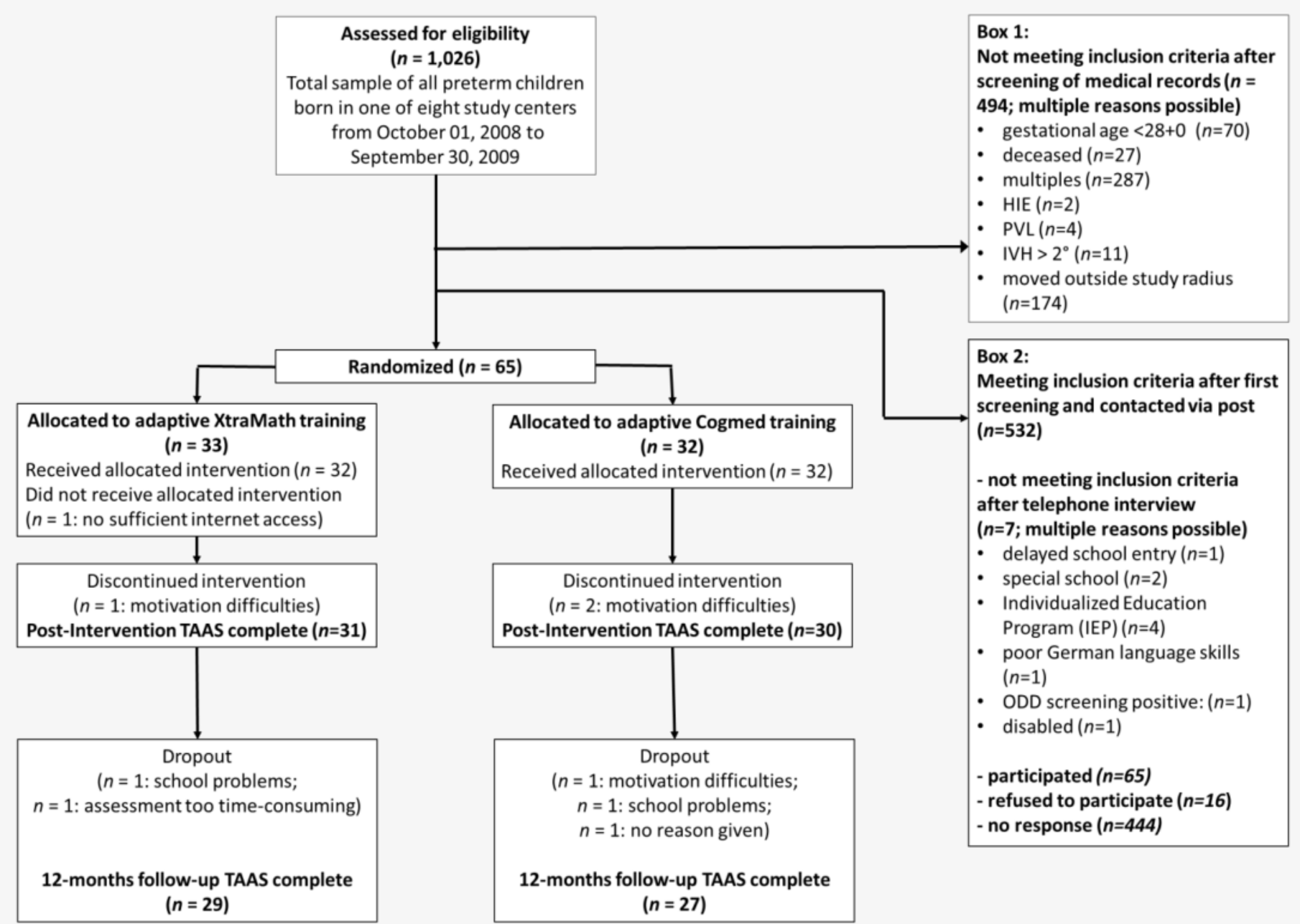

HIE: Hypoxic Ischemic Encephalopathy; PVL: Periventricular Leucomalacia; IVH: Intraventricular Hemorrhage; ODD: Oppositional Defiant Disorder
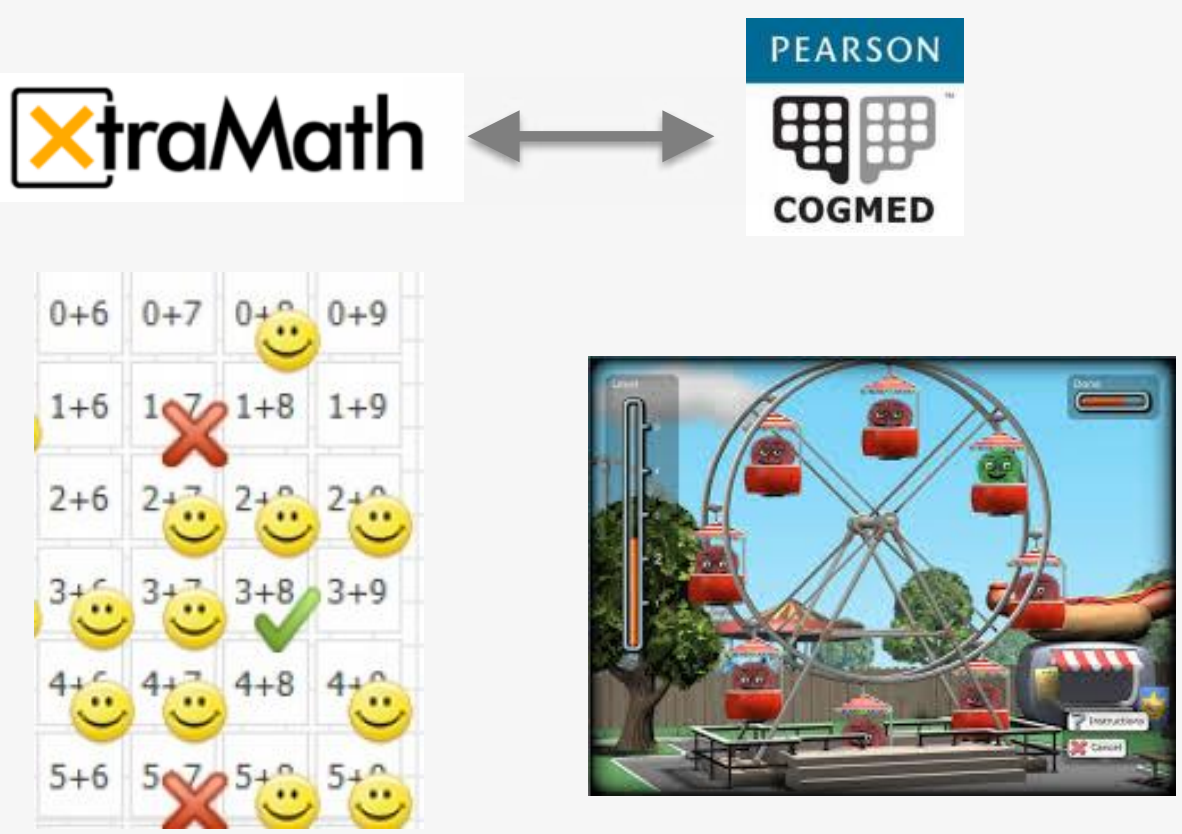

\section{Conclusion} mathematics.

\section{Results}

Bootstrapped linear regressions with intervention as main factor and baseline TAAS (T1) as covariate showed that total academic growth to post-test was higher in the XtraMath group $(\mathrm{B}=-.29$ [95\% Cl: -.60 to $.04], p=.045)$, but this difference was not sustained at 12 -months follow-up ( $B=.10$ [-.64 to .87], $p=.784$; Figure 2).

There were no significant group differences on secondary outcomes according to training condition, however, children in the XtraMath group showed a tendency to do better in math (Figure 3).

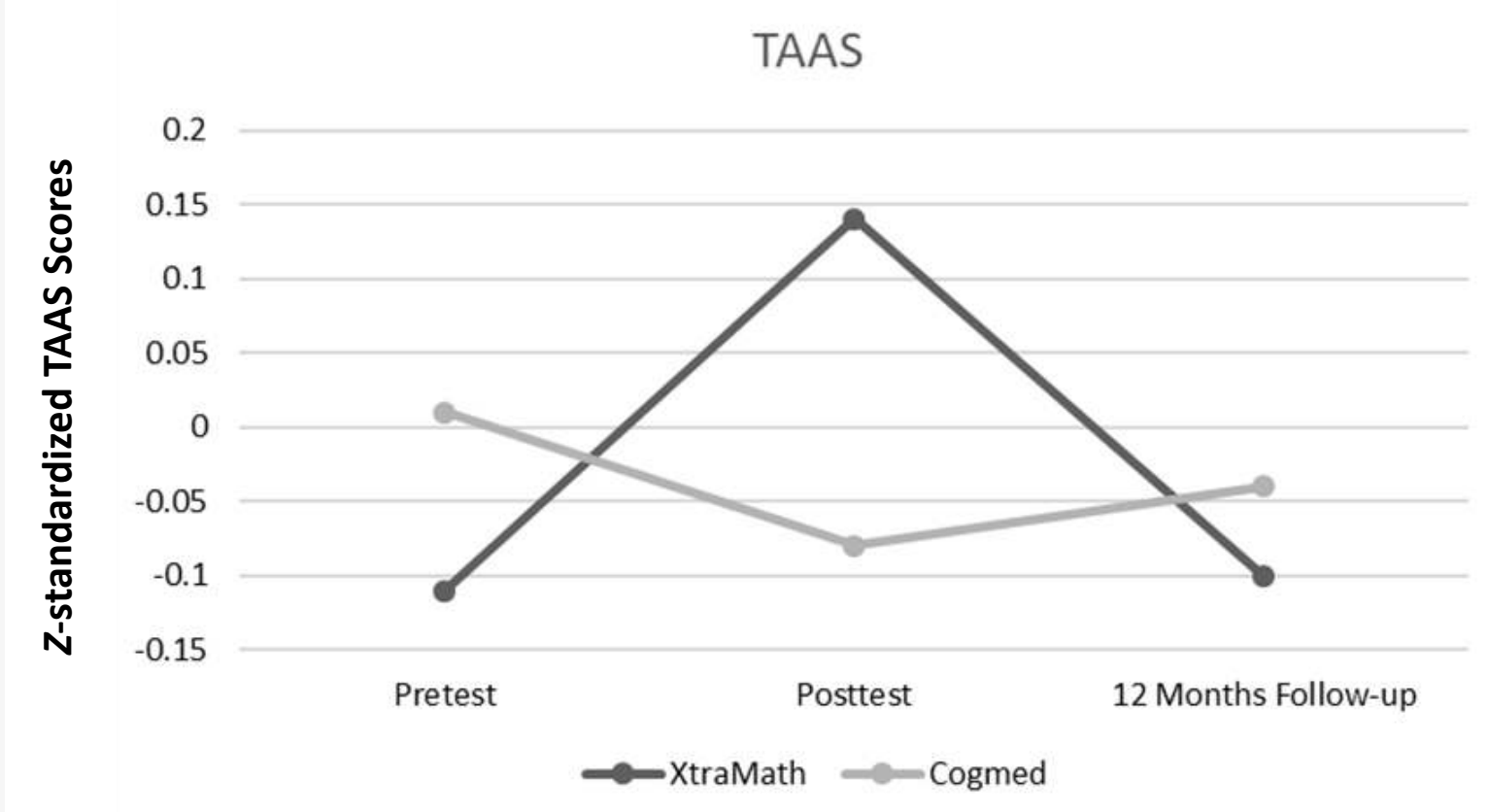

Figure 2. Teacher-rated Academic Attainment Score (TAAS) Growth by Intervention (XtraMath versus Cogmed)

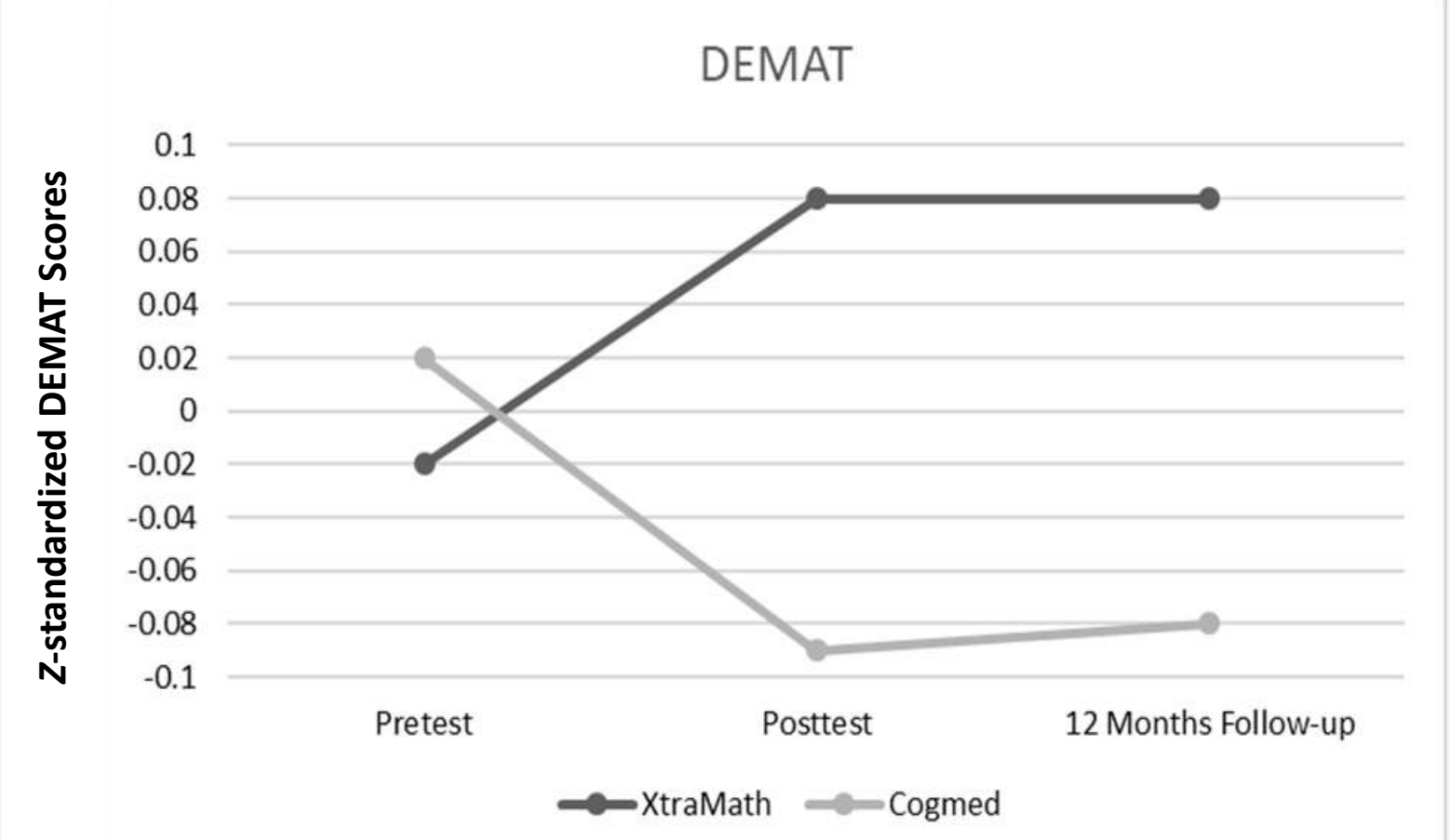

Figure 3. Standardized Math Test Scores (DEMAT) Growth by Intervention (XtraMath versus Cogmed)

Our findings do not show a difference in effectiveness between Cogmed and the open-access online training XtraMath, but XtraMath may tend to better support preterm children's success in

Please have a look at our related e-poster: Feasibility of adaptive computerized working memory and math intervention in 7-year-old children born preterm by Heuser et al.

Contact information: jjaekel@utk.edu 